\title{
Pathogenesis of seizures and epilepsy after stroke
}

\author{
Jiayu Chen, Haijiao Ye, Jie Zhang, Aihong Li * (D) and Yaohui Ni* (D)
}

\begin{abstract}
Stroke is the most frequent cause of secondary epilepsy in the elderly. The incidence of cerebral stroke is increasing with the extension of life expectancy, and the prevalence of post-stroke epilepsy (PSE) is rising. There are various seizure types after stroke, and the occurrence of epilepsy is closely related to the type and location of stroke. Moreover, the clinical treatment of post-stroke epilepsy is difficult, which increases the risk of disability and death, and affects the prognosis and quality of life of patients. Now seizure and epilepsy after stroke is more and more get the attention of the medical profession, has been more and more researchers have devoted to seizures after stroke and PSE clinical and basic research, and hope to get a scientific and unified guideline, to give timely and effective treatment, but the exact pathophysiologic mechanism has not yet formed a unified conclusion. It has been found that ion channels, neurotransmitters, proliferation of glial cells, genetics and other factors are involved in the occurrence and development of PSE. In this review, we discuss the pathogenesis of early-onset epileptic seizures and late-onset epilepsy after stroke, in order to provide a basis for clinicians to understand the disease, and expect to provide ideas for future exploration.
\end{abstract}

Keywords: Stroke, Epilepsy, Pathogenesis

\section{Background}

Epilepsy is the most common neurological disorder and a chronic brain disease with multiple causes. It is characterized by recurrent, paroxysmal, and transient central nervous system (CNS) dysfunction caused by excessive discharge of brain neurons. Genetic factors, abnormal labor (such as hypoxia, asphyxia, and head trauma during delivery), CNS infection, and anomalous brain development are important etiologies of seizures and epilepsy in children. Cerebrovascular disease, craniocerebral trauma, intracranial infection and metabolic abnormality are common causes of seizures and epilepsy in adults [1]. In the elderly, the most common cause of epilepsy is stroke [2]. With the extension of life expectancy, the incidence of stroke increases yearly and the number of patients with

*Correspondence: 15851205508@163.com; niyaohui200688@126.com Department of Neurology, Affiliated Hospital of Nantong University, Nantong University, Nantong 226001, Jiangsu, China post-stroke epilepsy (PSE) is also increasing. PSE refers to epilepsy that occurs after a hemorrhagic or ischemic stroke without a previous history of epilepsy, and is not accompanied by brain structural abnormalities or metabolic disorders of other causes. The PSE accounts for about $30-50 \%$ of newly diagnosed epilepsy [3]. A large population-based study has shown that in the first year after stroke, the risk of epilepsy is about 23 times higher than that in the general population [4]. The International League Against Epilepsy has divided PSE into early-onset epileptic seizures and late-onset epilepsy. The early epileptic seizures refer to seizures that occur within 1 week after stroke, mainly within $24 \mathrm{~h}$, accounting for about $57 \%$ of early epileptic seizures. Patients with early epileptic seizures do not form a stable epileptic network, and the brain has the ability to self-repair, so they cannot be diagnosed as epilepsy, but be diagnosed as seizures. The late-onset epilepsy occurs over 1 week after stroke, with highest incidence at 6-12 months after stroke. Ninety percent of patients may have seizures again after one 
attack $[5,6]$. Patients with late-onset epilepsy have definite epileptogenic foci and form a stable epileptic network, and are susceptible to recurrent seizures, thus they are diagnosed with epilepsy. PSE can aggravate disabilities, affect patients' quality of life and the outcome of the disease, and even lead to death. A comprehensive understanding of the pathogenesis of PSE is of great significance for the treatment and prevention of this disease. In the following, the pathogenesis of early epileptic seizures and late epilepsy after stroke will be discussed in detail.

\section{The pathogenic mechanisms of early-onset epileptic seizures \\ Ion channel dysfunction}

The stroke-induced acute ischemia and hypoxia can reduce the stability of nerve cell membrane and cause metabolic disorders of neuron. The acute brain injury can result in subsequent sodium pump failure, increased $\mathrm{Na}^{+}$ influx and depolarization of membrane potential. When the membrane's potential depolarization reaches a certain degree, calcium channels will be activated and $\mathrm{Ca}^{2+}$ rapidly flow in [7], leading to an increase in intracellular $\mathrm{Ca}^{2+}$ concentration. This results in overexcitation of neurons, causing excitatory toxicity and gradual loss of functions of local suppressor cells. When the local suppressor cells are unable to inhibit the spread of rhythmic, hypersynchronous discharges, epilepsy would occur [8].

\section{Neurotransmitter imbalance}

The early-onset epileptic seizures can also be caused by the disruption of the dynamic balance of neurotransmitters. The excitatory glutamate and the inhibitory $\gamma$-aminobutyric acid (GABA) are the most essential neurotransmitters in the CNS. Under physiological conditions, the excitatory and inhibitory inputs to cortical neurons are dynamically and proportionally balanced, which maintain stable neural networks. Studies have shown that due to ischemia and hypoxia of brain tissue after stroke, a large amount of glutamate is released, and post-synaptic glutamate receptors are over-activated, leading to increased neuronal excitability and a state of irritability of the brain, making the brain susceptible to epileptic seizures in patients $[9,10]$. In addition, after stroke, the mRNA and protein levels of GABA receptor $\alpha 1$ subunit are reduced, or the transport of GABA receptor to the cell membrane is reduced, which weakens the GABA receptor-dependent inhibitory postsynaptic potential and decreases the seizure threshold. Thus, seizure is easily induced [11, 12]. Other studies have shown that in the early stage of ischemic stroke, the level of GABA and the activity of GABA neurons decrease, resulting in an ionotropic glutamate $N$-methyl$D$-aspartic acid receptor (NMDAR)-mediated increase of nitric oxide (NO). The M-type $\mathrm{K}^{+}$channel is an important regulator of neuronal excitability; it can stabilize the resting membrane potential and affect the subthreshold excitability of inhibitory neurons. The increased NO can increase the excitability of the CNS by blocking the $\mathrm{M}$-type $\mathrm{K}^{+}$channels, causing epileptic seizures [13-16].

\section{Elevated serum cortisol levels}

In the early stages of ischemic stroke, the hypothalamicpituitary-adrenal (HPA) axis is activated in response to stress, which ignificantly increases the level of cortisol in blood [17] . Cortisol is neurotoxic; it can aggravate the hypoxic injury to neurons and astrocytes, and affect the uptake and metabolism of glucose in the brain, thus inducing epileptic seizures $[18,19]$. In addition, acute disorder with irritability is an independent predictnte glutamate receptors in the postsynaptic membrane of neurons, greatly increasing the excitability of the neurons [20]. Other studies have shown that after acute stroke, the high serum level of cortisol can also reduce the total number of nerve cells in the CA3 area of hippocampus and interfere with the neurogenesis of granulosa cells in adult rats, resulting in changes in temporal lobe structure and physiological function, leading to recurrent epileptic seizures in the temporal lobe [20,21]. In conclusion, in the early stage of acute stroke, the high serum level of cortisol is an important contributor to the onset of convulsion.

\section{Deposition of hemosiderin}

It is estimated that $4-16 \%$ of patients with hemorrhagic stroke will suffer epileptic seizure after intracerebral hemorrhage [22]. Studies have shown that hemosiderin deposition is closely related to the occurrence of early epileptic seizures after subarachnoid hemorrhage. Intracellular reactions induced by free radicals and free iron can contribute to the structural changes in brain regions surrounding the site of hemorrhage, which can cause synaptic recombination around the lesion and induce epileptic seizures. When hemosiderin accumulates locally in the gray matter of the brain, the iron released from the precipitated hemosiderin mainly exists in the form of ion, which would lead to production of hydroxyl radicals and affect the oxidative stress level of cortical neurons, leading to the occurrence of epilepsy [23].

In addition to the above mechanisms of early epileptic seizures, several other potential pathogenic mechanisms have been proposed, such as acute electrolyte disturbances (such as increased intracellular calcium and sodium concentrations) in the ischemic penumbra surrounding the stroke lesion, which lead to depolarization of neuronal membrane; increased concentration of extracellular glutamate; impaired function of GABAergic 
interneurons. This series of changes leads to the diffuse depolarization potentials in the ischemic penumbra. The depolarization potentials spreads from the ischemic penumbra to the ischemic core. Due to the continuous depolarization of neurons, epileptic seizures may occur [24]. There is also a possibility of ischemia-reperfusion injury caused by recanalization in the acute phase of ischemic stroke, and ischemic blood oxygen caused by local or diffuse vasospasm in hemorrhagic stroke. Ischemia and hypoxia can cause metabolic disorders of stroke-related neurons, increase neuronal excitability, and lead to eclampsia after stroke [10]. Studies have reported that the early epileptic seizures result in higher disability and mortality rates than late epilepsy [25]. In addition, people with advanced epilepsy have permanent structural changes in the brain. These structural changes cause neurons to fire abnormally and form fixed networks, which can lead to recurrent seizures [26].

\section{The pathogenic mechanisms of late epileptic seizures Astrocytic proliferation}

In later stages of stroke, the CNS is damaged, because the glial scar formed by reactive astrocytes can cause acquired epilepsies. The astrocytic formation of scar changes the physiological function of astrocytes, affecting the function of the neural network and leading to late epilepsy. Some studies have indicated that after stroke, upon acute brain injury, astrocytes far from the injury form long hypertrophic projections that grow toward the injury site. On the other hand, the protrusions formed by astrocytes in the core of the injury are arranged radially and evenly, overlap with each other, and form glial scars together [27]. The phenotypes of scar formation vary, mainly depending on the location of the injury. The morphological change of astrocytes after brain injury is relatively slow. Although the hypertrophic astrocytes can return back to a normal phenotype, the scar-forming astrocytes persistently express increased glial fibrin, even after the acute injury triggers disappear, therefore having permanent morphological changes [28]. Other studies have reported that astrocytes can release glutamate through Swell1 channels. In a study on the effects of glutamate release on neuronal excitability, mice with knockout of the Swell1 channel gene release less glutamate and thus have lower neuronal excitability than those without knockout [29]. After stroke, the increase of astrocytes lead to significantly increased release of glutamate through Swell1 channel, which has excitotoxicity effects on neurons and induce seizures. Other studies have shown that the increase of astrocytes is a common feature of late seizures after stroke. Astrocytes undergo morphological changes upon activation in many CNS diseases. Reactive astrocytes can be potential contributors to neuronal hyperexcitability and seizures. Reactive astrocytes have morphological and protein expression changes after stroke, while being deficient in physiological function, leading to impairment of glutamate homeostasis and increased production of excitatory neurotransmitter glutamate. At the same time, reactive astrocytes have impaired ability to absorb $\mathrm{K}^{+}$and decreased synthesis of inhibitory neurotransmitter GABA. The increase of glutamate and the decrease of GABA result in increased excitability of neurons and decreased threshold of seizures, leading to increased susceptibility to seizures. Reactive astrocytes also have changed composition of extracellular matrix, which indirectly affects synaptic function and neuronal homeostasis [30], thereby promoting the occurrence of epileptic seizures.

\section{Damage of blood-brain barrier (BBB)}

$\mathrm{BBB}$ refers to the barrier between plasma and brain cells formed by cerebral capillary wall and glial cells, and the barrier between plasma and cerebrospinal fluid formed by choroid plexus. These barriers can prevent the entry of some substances to the brain from blood. During an ischemic stroke, the BBB is destroyed, and the blood components seep into the brain tissue and impair neuronal function [31]. Cerebral ischemic injury can cause a late-onset vasogenic brain edema, which is related to the BBB damage [32,33]. BBB injury causes a flow of a large amount of blood-derived fluid to the extravascular space. Albumin is one of the most abundant proteins in blood, so one of the most obvious changes after BBB injury is the presence of albumin in brain tissue. In a mouse model of photothrombotic cortical stroke with cortical infarcted areas around the hippocampus, albumin extravasation was detected in the cortex, hippocampus, and some isolated cortical areas around the cerebral infarction. The extracellular albumin binds to the transforming growth factor $\beta$ (TGF $\beta$ ) receptor on astrocytes, activating TGF $\beta$ signaling in astrocytes, changing the gene expression of astrocytes and down-regulating the inward rectifier potassium (KIR4.1) channels and water (water channel 4) channels of astrocytes, which decreases the uptake of $\mathrm{K}^{+}$and glutamate by astrocytes. With the increase of extracellular $\mathrm{K}^{+}$and glutamate concentrations, the excitotoxicity of neurons increases and epileptic seizures are induced [34, 35]. Other studies have found that after stroke, the active components released from cells cause inflammation, resulting in damage to the integrity of BBB and increased permeability. Serine proteases (such as plasmin and thrombin) in blood infiltrate into the brain tissue, activate protease-activated receptor (PAR). the number of NMDARs has increased due to the activation of NMDARs, resulting in glutamate-mediated excitotoxicity [36]. In mice with pilocarpine-induced 
status epilepticus, the level of thrombin in hippocampal CA1 region increases significantly. Even a slight increase in thrombin can significantly increase the number of NMDARs, induce excitatory neurotoxicity and lead to seizures [37]. The increased expression of NMDAR can promote the formation of axons and the formation of new synapses. However, after stroke, a large number of PAR1 is activated, the NMDAR is overexpressed, and pathological synaptic reconstruction is activated, which lead to the reconstruction of neural network, and subsequent destruction of brain function and onset of seizure [26]. In a prospective study in 28 patients aged between 38 and 90 years with late-onset epilepsy after cortical stroke, brain DTPA-SPECT within $72 \mathrm{~h}$ of the first seizure showed BBB disruption in the stroke area in $85.7 \%$ of the patients with late-onset epilepsy after cortical stroke, compared to $28.6 \%$ of patients without epilepsy after cortical stroke [38]. Recent studies have also shown that the disruption of BBB is the cause of seizures and has become an important factor in the formation of epileptic foci or epileptogenic foci [39].

\section{Genetics and genes}

About $30 \%$ of all epileptic syndromes are hereditary, and more than 500 genetic loci have been revealed to be associated with epilepsy in humans and mice [40]. Some studies have discussed the relationship between heredity and PSE and found that a polymorphism of the rs671 allele encoding mitochondrial acetaldehyde dehydrogenase 2 (ALDH2) is associated with late post-stroke seizures. Patients with the rs671 allele polymorphism have higher levels of 4-hydroxy-2-nonanal (4-HNE), a specific marker of oxidative stress. The level of 4-HNE is significantly increased in genetically susceptible stroke rats, experimental rats with middle cerebral artery occlusion, and patients with epilepsy after stroke. The increase of 4-HNE is related to epilepsy in the late stage of ischemic stroke. ALDH2 metabolizes 4-HNE to acetic acid and detoxifies aldehydes produced by reactive oxygen species to reduce neuronal apoptosis [41]. Accumulating evidence has shown that oxidative stress caused by the increase of reactive oxygen species or nitrogen is related to the occurrence and development of epilepsy [42]. In another study on the relationship between CD40 functional polymorphism and susceptibility to epilepsy after stroke, the expression of CD40 mRNA and protein in peripheral blood mononuclear cells of PSE patients was significantly higher than that of normal control patients with ischemic stroke. In addition, in patients with late epilepsy after stroke, the plasma sCD40L level and CD40 mRNA expression were increased in T-allele carriers. This study suggests that the CD40-1C/T allele polymorphism is related with the susceptibility to epilepsy after stroke [43]. In addition, in a study on whether the functional polymorphism of TRPM6 (rs2274924) is related to the susceptibility to epilepsy after ischemic stroke, three genotypes of TRPM6 rs2274924 were identified by DNA sequencing: TT, CT and CC. The frequency of CC genotype in patients with PSE was significantly higher than that in the control group without epilepsy, and the distribution of rs2274924C allele was wider in patients with PSE. The $C$ allele of rs2274924 is related to low serum levels of $\mathrm{Mg}^{2+}$, increase the intracellular $\mathrm{Na}^{+}$, promote the exchange of sodium/calcium, increase the concentration of intracellular $\mathrm{Ca}^{2+}$, increase the excitability of neurons, and induce seizures. It is inferred that the polymorphism of TRPM6 rs2274924 and the low level of serum $\mathrm{Mg}^{2+}$ are potential predictors of epilepsy after stroke [44].

\section{Structural changes of brain network}

With development of electrophysiological and neuroimaging studies on the structure and function of the brain, epilepsy is increasingly considered a whole functional disorder of the brain. In recent years, the mechanism of epilepsy has been gradually explained from the perspective of neural networks. Susan S. Spencer defined the epileptic network between anatomy and function of the cerebral cortex and subcutaneous structures. Abnormal activity of any part of the network will affect the activity of all other parts [45]. Late epilepsy after stroke, as typical focal seizures, are also related to changes in neural networks. Some studies have shown that ischemia and hypoxia can lead to massive neuronal loss and damage, but the brain still has a certain ability of endogenous regeneration to generate new neurons, which can then be integrated into primitive neuronal pathways [46]. Abnormal discharge of the newly generated neural networkcan cause seizures. As an in vitro model of acquired epilepsy caused by stroke, hippocampal neurons were cultured with different concentrations of glutamate for different durations. Glutamate induced death or stroke-like injury in neuronal subsets; some neurons die from excitotoxicity, while more neurons survive after injury, similar to those in the ischemic penumbra. The surviving neurons show spontaneous, recurrent, epileptic discharges in the neural network [47]. In addition, in thrombin-focused animal models, the PAR pathway can be considered as the central hub of this regulatory brain network, mainly through thrombin or activated protein $\mathrm{C}$. These proteins can interfere with synaptic homeostasis and affect synaptic plasticity, thus regulating neural networks and causing seizures [48]. In addition, in 7-month-old mice which suffered neonatal strokes caused by unilateral carotid ligation, the induction of activity-regulated cytoskeleton associated protein (Arc) in hippocampal neurons was impaired in the bilateral hippocampi, indicating that the 
bilateral functional hippocampal circuits are activated by joint input from the cerebral hemisphere. In addition, the epilepsy after stroke may stimulate neurogenesis on the contralateral side, thus increasing the susceptibility of neural network to epilepsy [49]. Mossy fiber sprouting in the hippocampus can be observed at the site of acquired epilepsy, and can be observed in both the diseased side and the non-diseased hemisphere. However, there are relatively few studies on the changes of epileptic functional network after stroke. Further research is needed to clarify the relationship between neural networks and PSE.

\section{Regional hemodynamic changes}

Local changes in cerebral blood hemodynamics may also induce seizures. In a study of local cerebral blood flow in patients with persistent psycho-behavioral changes after epileptic seizures, there were significant increases in local cerebral blood flow and volume in 9 patients with nonconvulsive continuous state seizures, compared with 10 patients with postictal state [50]. In another study, 78.9\% of patients with PSE had regional cortical hyperperfusion at the onset, with significantly increased local cerebral blood flow (rCBF) values in the affected and unaffected cortical areas. Cerebral perfusion CT at 90 days of followup showed that 8 of the 9 patients had decreased intensity of high-perfusion area and $\mathrm{rCBF}$ value, which suggested that some epileptic seizures may be caused by changes in local cerebral perfusion [51]. Another study showed that the incidence of epileptic seizures after thrombolysis is $4-15 \%$, the incidence of early seizures is $2.5-5 \%$, and the incidence of late seizures is $1.5-11.3 \%$, higher than the incidence of PSE without thrombolysis. In addition, epileptic seizures during or after thrombolysis may be a sign of successful reperfusion, thus the local hemodynamic changes caused by reperfusion therapy are risk factors for epileptic seizures [52].

\section{Conclusions}

Stroke is the most common cause of acquired epilepsy. The pathogenesis of epilepsy after stroke has been studied in depth, with some mechanisms confirmed while others being new hypotheses that awaits confirmation. Currently, it is widely accepted that epilepsy occurs after stroke due to the imbalance of excitatory and inhibitory transmission in the CNS. Some mechanisms have also been proposed, including metabolic abnormalities, synaptic reorganization, proliferation of astrocytes, destruction of BBB, genetic susceptibility and changes in brain network structure. In particular, the changes of brain network structure in patients with epilepsy after stroke are receiving more and more attention, and further studies are needed to determine the independent predictors of post-stroke seizures and different types of seizures, in order to guide clinical prediction of stroke patients who may develop some type of epilepsy. It is important to investigate the new brain network structure in patients with epilepsy after stroke to determine whether there is a new fixed epileptogenic network. This will provide insight into targeted drug intervention strategies in patients with specific brain network structure to prevent the onset of epilepsy and ameliorate aggravation of disability in patients with stroke.

\section{Abbreviations \\ PSE: Post-stroke epilepsy; GABA: Glutamate and $\gamma$-aminobutyric acid; NMDAR: $\mathrm{N}$-methyl-D-aspartic acid receptor; NO: Nitric oxide; HPA: Hypothalamic-pitui- tary-adrenal; BBB: Blood-brain barrier; TGF- $\beta$ : Transforming growth factor $\beta$; PAR: Protease-activated receptor; ALDH2: Acetaldehyde dehydrogenase 2; 4-HNE: 4-hydroxy-2-nonanal; Arc: Activity-regulated cytoskeleton associated protein.}

\section{Acknowledgements \\ Not applicable.}

\section{Authors' contributions}

AL and YN conceptualized and revised the article. JC searched and read the articles related to seizures and epilepsy after stroke, summarized the views and wrote the manuscript. HY and JZ made supplementary modifications to the article. All authors read and approved the final manuscript.

\section{Funding}

None.

\section{Availability of data and materials}

Not applicable.

\section{Declarations}

Ethics approval and consent to participate Not applicable.

\section{Consent for publication}

Not applicable.

\section{Competing interests}

The authors declare no financial or other conflicts of interest.

Received: 23 May 2021 Accepted: 2 December 2021

Published online: 04 January 2022

\section{References}

1. Beghi E. The epidemiology of epilepsy. Neuroepidemiology. 2020;54(2):185-91.

2. Sen A, Jette N, Husain M, Sander JW. Epilepsy in older people. Lancet. 2020;395(10225):735-48.

3. Zhao Y, Li X, Zhang K, Tong T, Cui R. The Progress of epilepsy after stroke. Curr Neuropharmacol. 2018;16(1):71-8.

4. So EL, Annegers JF, Hauser WA, O'Brien PC, Whisnant JP. Populationbased study of seizure disorders after cerebral infarction. Neurology. 1996;46(2):350-5.

5. Yang H, Rajah G, Guo A, Wang Y, Wang Q. Pathogenesis of epileptic seizures and epilepsy after stroke. Neurol Res. 2018;40(6):426-32.

6. Holtkamp M, Beghi E, Benninger F, Kalviainen R, Rocamora R, Christensen $\mathrm{H}$, et al. European stroke organisation guidelines for the management of post-stroke seizures and epilepsy. Eur Stroke J. 2017;2(2):103-15. 
7. Denier C, Masnou P, Mapoure Y, Souillard-Scemama R, Guedj T, Theaudin $\mathrm{M}$, et al. Watershed infarctions are more prone than other cortical infarcts to cause early-onset seizures. Arch Neurol. 2010;67(10):1219-23.

8. Chung JM. Seizures in the acute stroke setting. Neurol Res. 2014;36(5):403-6.

9. Mizielinska SM, Greenwood SM, Tummala H, Connolly CN. Rapid dendritic and axonal responses to neuronal insults. Biochem Soc Trans. 2009;37(Pt 6):1389-93.

10. Sun DA, Sombati S, DeLorenzo RJ. Glutamate injury-induced epileptogenesis in hippocampal neurons: an in vitro model of stroke-induced "epilepsy". Stroke. 2001;32(10):2344-50.

11. Kessler KR, Schnitzler A, Classen J, Benecke R. Reduced inhibition within primary motor cortex in patients with poststroke focal motor seizures. Neurology. 2002;59(7):1028-33.

12. Kharlamov EA, Downey KL, Jukkola PI, Grayson DR, Kelly KM. Expression of GABA a receptor alpha1 subunit $\mathrm{mRNA}$ and protein in rat neocortex following photothrombotic infarction. Brain Res. 2008;1210:29-38.

13. Bierbower SM, Choveau FS, Lechleiter JD, Shapiro MS. Augmentation of M-type (KCNQ) potassium channels as a novel strategy to reduce strokeinduced brain injury. J Neurosci. 2015:35(5):2101-11.

14. Ooi L, Gigout S, Pettinger L, Gamper N. Triple cysteine module within M-type $K+$ channels mediates reciprocal channel modulation by nitric oxide and reactive oxygen species. J Neurosci. 2013;33(14):6041-6.

15. Delmas P, Brown DA. Pathways modulating neural KCNQ/M (Kv7) potassium channels. Nat Rev Neurosci. 2005;6(11):850-62.

16. Hosinian M, Qujeq D, Ahmadi AA. The relation between GABA and L-arginine levels with some stroke risk factors in acute ischemic stroke patients. Int J Mol Cell Med. 2016;5(2):100-5.

17. Fassbender K, Schmidt R, Mossner R, Daffertshofer M, Hennerici M. Pattern of activation of the hypothalamic-pituitary-adrenal axis in acute stroke. Relation to acute confusional state, extent of brain damage, and clinical outcome. Stroke. 1994;25(6):1105-8.

18. Virgin CE Jr, Ha TP, Packan DR, Tombaugh GC, Yang SH, Horner HC, et al. Glucocorticoids inhibit glucose transport and glutamate uptake in hippocampal astrocytes: implications for glucocorticoid neurotoxicity. J Neurochem. 1991;57(4):1422-8.

19. Tombaugh GC, Yang SH, Swanson RA, Sapolsky RM. Glucocorticoids exacerbate hypoxic and hypoglycemic hippocampal injury in vitro: biochemical correlates and a role for astrocytes. J Neurochem. 1992;59(1):137-46.

20. Kanner AM, Scharfman H, Jette N, Anagnostou E, Bernard C, Camfield C, et al. Epilepsy as a network disorder (1): what can we learn from other network disorders such as autistic spectrum disorder and mood disorders? Epilepsy Behav. 2017;77:106-13.

21. Rajkowska G, Miguel-Hidalgo JJ, Wei J, Dilley G, Pittman SD, Meltzer $H Y$, et al. Morphometric evidence for neuronal and glial prefrontal cell pathology in major depression. Biol Psychiatry. 1999:45(9):1085-98.

22. Conrad J, Pawlowski M, Dogan M, Kovac S, Ritter MA, Evers S. Seizures after cerebrovascular events: risk factors and clinical features. Seizure. 2013;22(4):275-82.

23. Zhang L, Zou X, Zhang B, Cui L, Zhang J, Mao Y, et al. Label-free imaging of hemoglobin degradation and hemosiderin formation in brain tissues with femtosecond pump-probe microscopy. Theranostics. 2018:8(15):4129-40.

24. Gavaret M, Marchi A, Lefaucheur JP. Clinical neurophysiology of stroke. Handb Clin Neurol. 2019;161:109-19.

25. Bladin CF, Alexandrov AV, Bellavance A, Bornstein N, Chambers B, Cote R, et al. Seizures after stroke: a prospective multicenter study. Arch Neurol. 2000;57(11):1617-22

26. Altman K, Shavit-Stein E, Maggio N. Post stroke seizures and epilepsy: from proteases to maladaptive plasticity. Front Cell Neurosci. 2019;13:397.

27. Oberheim NA, Tian GF, Han X, Peng W, Takano T, Ransom B, et al. Loss of astrocytic domain organization in the epileptic brain. J Neurosci. 2008;28(13):3264-76.

28. Robel S, Berninger B, Gotz M. The stem cell potential of glia: lessons from reactive gliosis. Nat Rev Neurosci. 2011;12(2):88-104.

29. Yang J, Vitery MDC, Chen J, Osei-Owusu J, Chu J, Qiu Z. Glutamate-releasing SWELL1 channel in astrocytes modulates synaptic transmission and promotes brain damage in stroke. Neuron. 2019;102(4):813-27 e6.

30. Robel S. Astroglial scarring and seizures: a cell biological perspective on epilepsy. Neuroscientist. 2017;23(2):152-68.
31. Jiang $X$, Andjelkovic AV, Zhu L, Yang T, Bennett MVL, Chen J, et al. Blood-brain barrier dysfunction and recovery after ischemic stroke. Prog Neurobiol. 2018:163-164:144-71.

32. Stokum JA, Gerzanich V, Simard JM. Molecular pathophysiology of cerebral edema. J Cereb Blood Flow Metab. 2016;36(3):513-38.

33. Dharmasaroja PA. Fluid intake related to brain edema in acute middle cerebral artery infarction. Transl Stroke Res. 2016;7(1):49-53.

34. David Y, Cacheaux LP, Ivens S, Lapilover E, Heinemann U, Kaufer D, et al. Astrocytic dysfunction in epileptogenesis: consequence of altered potassium and glutamate homeostasis? J Neurosci. 2009;29(34):10588-99.

35. van Vliet EA, Aronica E, Gorter JA. Blood-brain barrier dysfunction, seizures and epilepsy. Semin Cell Dev Biol. 2015;38:26-34.

36. Semenikhina M, Bogovyk R, Fedoriuk M, Nikolaienko O, Al Kury LT, Savotchenko A, et al. Inhibition of protease-activated receptor 1 ameliorates behavioral deficits and restores hippocampal synaptic plasticity in a rat model of status epilepticus. Neurosci Lett. 2019;692:64-8.

37. Isaev D, Lushnikova I, Lunko O, Zapukhliak O, Maximyuk O, Romanov A, et al. Contribution of protease-activated receptor 1 in status epilepticusinduced epileptogenesis. Neurobiol Dis. 2015;78:68-76.

38. Gilad R, Lampl Y, Eilam A, Boaz M, Loyberboim M. SPECT-DTPA as a tool for evaluating the blood-brain barrier in post-stroke seizures. J Neurol. 2012;259(10):2041-4

39. Tanaka T, Ihara M. Post-stroke epilepsy. Neurochem Int. 2017;107:219-28.

40. Pitkanen A, Roivainen R, Lukasiuk K. Development of epilepsy after ischaemic stroke. Lancet Neurol. 2016;15(2):185-97.

41. Yang $H$, Song Z, Yang GP, Zhang BK, Chen M, Wu T, et al. The ALDH2 rs671 polymorphism affects post-stroke epilepsy susceptibility and plasma 4-HNE levels. PLoS One. 2014;9(10):e109634.

42. Folbergrova J, Otahal J, Druga R. Brain superoxide anion formation in immature rats during seizures: protection by selected compounds. Exp Neurol. 2012:233(1):421-9.

43. Zhang B, Chen M, Yang H, Wu T, Song C, Guo R. Evidence for involvement of the CD40/CD40L system in post-stroke epilepsy. Neurosci Lett. 2014:567:6-10

44. Fu CY, Chen SJ, Cai NH, Liu ZH, Zhang M, Wang PC, et al. Increased risk of post-stroke epilepsy in Chinese patients with a TRPM6 polymorphism. Neurol Res. 2019;41(4):378-83.

45. Spencer SS. Neural networks in human epilepsy: evidence of and implications for treatment. Epilepsia. 2002;43(3):219-27.

46. Kadam SD, Mulholland JD, McDonald JW, Comi AM. Neurogenesis and neuronal commitment following ischemia in a new mouse model for neonatal stroke. Brain Res. 2008:1208:35-45.

47. DeLorenzo RJ, Sun DA, Blair RE, Sombati S. An in vitro model of strokeinduced epilepsy: elucidation of the roles of glutamate and calcium in the induction and maintenance of stroke-induced epileptogenesis. Int Rev Neurobiol. 2007:81:59-84

48. De Luca C, Virtuoso A, Maggio N, Papa M. Neuro-coagulopathy: blood coagulation factors in central nervous system diseases. Int J Mol Sci. 2017:18(10):2128.

49. Kadam SD, Smith-Hicks CL, Smith DR, Worley PF, Comi AM. Functional integration of new neurons into hippocampal networks and poststroke comorbidities following neonatal stroke in mice. Epilepsy Behav. 2010:18(4):344-57.

50. Hauf M, Slotboom J, Nirkko A, von Bredow F, Ozdoba C, Wiest R. Cortical regional hyperperfusion in nonconvulsive status epilepticus measured by dynamic brain perfusion CT. AJNR Am J Neuroradiol. 2009;30(4):693-8.

51. Gonzalez-Cuevas M, Coscojuela P, Santamarina E, Pareto D, Quintana M, Sueiras $\mathrm{M}$, et al. Usefulness of brain perfusion $\mathrm{CT}$ in focal-onset status epilepticus. Epilepsia. 2019;60(7):1317-24.

52. Brondani R, de Almeida AG, Cherubini PA, Secchi TL, de Oliveira MA, Martins SCO, et al. Risk factors for epilepsy after thrombolysis for ischemic stroke: a cohort study. Front Neurol. 2019:10:1256. 